\section{Organisations professionnelles}

\section{Rapprochement entre la FNCG et Fedalim}

La FNCG, Fédération nationale des industries de corps Gras (qui rassemble les familles professionnelles des huileries, de la margarine, des bougies, des corps gras animaux, du savon et de la détergence) et Fedalim, pôle de regroupement de sept organisations professionnelles de l'industrie alimentaire*, se sont rapprochées depuis le 10 avril dernier. Leurs tailles voisines, leurs valeurs communes (primauté des métiers, gouvernance fédérale, communauté d'intérêts des entreprises quelle que soit leur taille) ont permis d'envisager ce rapprochement vers un modèle d'organisation professionnelle à la fois léger, flexible et efficace.

En pratique, FNCG et Fedalim conservent leur indépendance politique et leur ancrage métier respectif. Les deux fédérations sont localisées ensemble au 66, rue La Boétie, Paris $8^{\mathrm{e}}$ et mettent en commun leurs compétences, leurs moyens et leurs équipes au service de leurs adhérents. Hubert Bocquelet est nommé délégué général des deux organisations; Fedalim est présidée par Dominique Amirault et la FNCG par Yves Delaine.

*Les sept organisations professionnelles de Fedalim :

STEPI, Syndicat du Thé et des Plantes à Infusion.

SNPE, Syndicat National des transformateurs de Poivres, Epices, aromates et vanille.

SYMTIA, Syndicat national des Mélanges Technologiques pour l'Industrie Alimentaire.

SCF, Syndicat de la Chicorée de France.

FNTPT, Fédération Nationale des Transformateurs de Pommes de Terre.

SNFBP, Syndicat National des Fabricants de Bouillons et Potages.

FICF, Fédération des Industries Condimentaires de France.

\section{Lipides et nutrition}

\section{Appel à projets GLN 2014}

Le GLN, Groupe Lipides et Nutrition, lance son appel à candidature 2014 pour le financement de projets de recherche clinique ou fondamentale sur la thématique Lipides et nutrition. Cette année, une attention particulière, mais non exclusive, sera portée sur l'acide alpha-linolénique (consommation, biodisponibilité, métabolisme, aspects mécanistiques...).

Rappelons que l'objectif du GLN est de favoriser l'étude des problèmes scientifiques et techniques relatifs aux corps gras alimentaires, afin de contribuer aux progrès des connaissances sur leur valeur nutritionnelle et leurs conditions d'utilisation par l'organisme humain. L'association réunit chercheurs et techniciens du secteur public et du secteur privé travaillant dans le domaine des lipides, et constitue un lieu de rencontre entre scientifiques et industriels.

En pratique, la date limite des dépôts de dossiers est fixée au vendredi 3 octobre 2014. Les dossiers sont à retirer auprès du GLN : gln@fncg.fr.

\section{Chimie verte}

\section{Une avancée pour améliorer la production de lipides chez la levure}

Principale forme de stockage de lipides, les triglycérides jouent un rôle majeur dans le développement des végétaux. Leur synthèse met en jeu trois étapes dont la dernière, décisive, fait intervenir une famille d'enzymes : les diacylglycérol acyltransférases (DGAT). Il existe plusieurs formes : DGAT1 qui est associée aux huiles alimentaires, DGAT2 l'est plutôt aux huiles industrielles. Les DGAT2 semblent essentielles pour l'accumulation de lipides d'intérêt utilisés, par exemple, dans la formulation de peintures et de vernis (notamment l'acide ricinoléique du ricin).

Pour la première fois, grâce à une stratégie d'optimisation de l'utilisation du code génétique, une équipe menée par l'Inra Versailles-Grignon, en collaboration avec le CNRS et l'Iterg, a réussi à exprimer DGAT2 de manière active dans une levure. Les chercheurs ont montré que l'expression du gène DGAT2 d'Arabidopsis thaliana restaure l'accumulation des triglycérides chez une levure incapable de les accumuler. Ils ont également mis en évidence que l'expression de DGAT (1 et 2) chez la levure induit la formation de vésicules lipidiques. Si ces dernières renferment des triglycérides, elles contiennent aussi du squalène, une molécule aux applications biotechnologiques potentielles (cosmétologie, compléments alimentaires) qui, à l'heure actuelle, est essentiellement prélevée sur des foies de requins.

La stratégie d'expression mise en œuvre par les scientifiques indique que l'expression de DGAT (1 et 2) est contrôlée par un usage subtil du code génétique, légèrement différent entre la plante et la levure. Cette technique se révèle être un outil précieux pour exprimer la DGAT et augmenter ainsi l'accumulation de lipides dans la levure.

Dans le contexte économique et écologique actuel de réduction de l'utilisation des produits issus du pétrole ou d'origine animale, l'élaboration de lipides par des microorganismes 
est en plein essor. Ces enzymes DGAT (1 et 2) pourraient ainsi devenir des cibles d'intérêt majeur pour la modification des voies métaboliques de biosynthèse des huiles (agroalimentaires et industrielle) et pour l'amélioration de leur production.

Ce travail a été réalisé dans le cadre du projet Carburants pour l'aéronautique, coordonné par l'Institut français du pétrole Énergies nouvelles. Il fait partie intégrante de la thèse «Contrat jeune scientifique » Inra de Laure Aymé.

Référence : Aymé L, Baud S, Joffre F, Dubreucq B, Chardot T. 2014. Function and localization of the Arabidopsis thaliana diacylglycerol acyltransferase DGAT2 expressed in yeast. PLoS One, 24 mars 2014. http://dx.plos.org/10.1371/ journal.pone.0092237.

\section{Méthanisation}

\section{L'Iterg, partenaire du projet européen Biogas}

L'Iterg est partenaire du projet européen Biogas, coordonné par l'Ainia (centre technologique espagnol). Le projet a débuté en mars 2014 pour une durée de deux ans. Il a pour objectif de promouvoir la production d'énergie renouvelable directement sur site industriel par l'utilisation de méthaniseurs de petites puissances qui traiteraient les résidus organiques des agro-industries.

Il consiste à :

- faire le bilan des résidus et coproduits organiques générés par les agro-industries et de leurs besoins énergétiques,

- mettre à jour et adapter aux pays membres du projet un logiciel permettant d'évaluer la faisabilité économique de l'installation de méthaniseurs de petites puissances sur les sites industriels,

- lister les fournisseurs d'installations de méthanisation adaptées aux industries agro-alimentaires,

- tester ce logiciel et réaliser une analyse de faisabilité avec des industriels souhaitant mettre en œuvre ce type de solution,

- sensibiliser les industriels à cette technologie (organisation de réunion d'information, de visite d'installation, etc.).

Ce projet fait suite à VECIR, achevé en 2011, et qui avait pour objectif de développer une voie de méthanisation pour le traitement des co-produits issus du secteur des huiles végétales. Différents essais de co-méthanisation des co-produits de l'huilerie avaient été réalisés et avaient permis de définir l'aptitude à la méthanisation de onze catégories de co-produits de l'huilerie.

\section{Synthèse}

\section{L'agriculture climato-intelligente}

L'agriculture climato-intelligente (ACI) repose sur trois piliers : la sécurité alimentaire et le bien-être des agriculteurs, l'adaptation au changement climatique, l'atténuation du changement climatique. L'ACI est un moyen pour mettre en oeuvre des priorités de développement agricole justifiées par le changement climatique à court et à long terme ; elle peut aussi servir à intégrer d'autres priorités de développement. Elle devra aider les pays et les divers acteurs à mettre en place les conditions politiques, techniques et financières qui leur permettront :

- d'augmenter durablement la productivité et les revenus agricoles ;

- de renforcer la résilience et la capacité des systèmes agricoles et alimentaires à s'adapter au changement climatique ;

- de réduire et d'éliminer les émissions de gaz à effet de serre (GES) tout en atteignant les objectifs nationaux de sécurité alimentaire et de développement.

Une note a été rédigée par une groupe de chercheurs du Cirad, de l'université de Wageningen (WUR), de la Banque mondiale, de l'université de Californie à Davis, de la FAO, $\mathrm{du}$ Conseil pour la recherche scientifique et industrielle du Ghana, et du programme Climate Change, Agriculture and Food Security du CGIAR (CCAFS). Elle est téléchargeable (PDF) sur : http://www.cirad.fr, Accueil > Publications \& ressources $>$ Edition $>$ Etudes et documents $>$ L'agriculture climato-intelligente.

\section{Recherche}

\section{Le génome d'un trypanosome des palmiers vient d'être séquencé}

Une collaboration sur un projet ANR entre l'équipe CEA du Génoscope dirigée par Patrick Wincker, l'équipe de Michel Dollet au Cirad et des laboratoires européens et américains vient de permettre le séquençage des génomes d'un trypanosome (parasite) responsable de dépérissements subits du cocotier et du palmier à huile en Amérique Latine et dans le sud de la Caraibe et d'autre part d'un trypanosome d'euphorbe non pathogène du sud de la France. Les travaux ont été publiés dans la revue PLoS Genetics de février 2014 (DOI : 10.1371/journal.pgen. 1004007).

Ce projet concernait un trypanosome quasi-symbionte que l'on trouve dans des euphorbes à Montpellier et un pathogène (associé au «Hartrot» du cocotier en Guyane) qui peut détruire une petite cocoteraie ou palmeraie en moins de 5 ans. Le séquençage s'est effectué au Génoscope et l'annotation a été réalisée par un consortium regroupant 25 chercheurs de 8 pays appartenant à 16 institutions.

Les trypanosomes sont surtout connus comme agents pathogènes de l'homme et de l'animal, provoquant de graves maladies comme la maladie du sommeil (Trypanosoma spp.), la maladie de Chagas (Trypanosoma cruzi) et les leishmanioses (Leishmania spp.). Leur génome a été révélé en 2005. La comparaison des séquences de ces deux trypanosomatides entre elles et avec celles de trypanosomes pathogènes de l'homme, révèle deux génomes distinctifs très compacts - « réduits au minimum »-, consistant essentiellement en copies uniques de gènes et une large majorité des gènes est partagée par les deux isolats indépendamment de leur effet sur l'hôte (pathogène ou quasi-symbionte). Ils possèdent beaucoup moins de gènes que les trypanosomes qui infectent les animaux. Une cohorte importante d'enzymes impliquées dans le métabolisme des sucres semble en adéquation avec les milieux (sève ou tube laticifère) dans lesquels ils évoluent. 


\section{Karité au Burkina Faso}

\section{Moins d'énergie pour un meilleur revenu et une filière durable}

Au Burkina Faso comme dans les autres pays d'Afrique subsaharienne, le beurre de karité est exploité pour le marché domestique et l'exportation. Son extraction à partir de l'amande des fruits est réalisée par les femmes selon une méthode qui demande beaucoup de main-d'œuvre et d'énergie. C'est une filière traditionnelle, qui obéit peu à des critères économiques (fort rôle social de l'activité, utilisation de ressources domestiques non marchandes...), mais doit malgré tout faire preuve de compétitivité pour générer des revenus. Pour l'insérer dans un marché concurrentiel, il n'est pas question de moderniser et de mécaniser les processus de production en imposant des technologies difficilement appropriables par les productrices, mais plutôt d'améliorer l'efficacité des procédés traditionnels.

Les chercheurs du Cirad s'intéressent à cette filière depuis plusieurs années. Cette nouvelle étude étude s'appuie sur un travail d'enquête et d'observation des pratiques traditionnelles de plusieurs groupements de femmes cueilleuses de fruits de karité et productrices de beurre dans plusieurs villages de la région est du Burkina Faso. Elle a permis de mesurer précisément les flux énergétiques et financiers occasionnés par les processus de production actuels ainsi que les prélèvements sur l'environnement. Elle a aussi permis de simuler l'introduction de nouvelles techniques (foyer amélioré en banco et séchoir coquillage pour la stabilisation et le séchage des fruits lors de la collecte, foyer amélioré métallique et torréfacteur à gaz butane pour la transformation) et de nouvelles pratiques (utilisation des coques et des boues de barattage comme combustibles) selon plusieurs scénarios combinant ces propositions.

Pour produire $1 \mathrm{~kg}$ de beurre, il faut actuellement 7,9 kg de bois de feu. En combinant les alternatives proposées, les productrices peuvent complètement se passer de bois en utilisant comme combustibles les coques, les boues de barattage séchées et un peu de butane (pour l'étape de torréfaction). Elles réduisent ainsi considérablement la pénibilité de leur travail et voient leurs bénéfices passer de 47 à 83 FCFA par kilogramme de fruit traité, et de 239 à 343 FCFA par kilogramme d'amandes transformées en beurre. Au total, le coût de l'énergie chute de $31 \%$ à $6 \%$ du coût de production du beurre. Ces travaux se sont limités à mesurer l'intérêt d'utiliser autrement l'énergie pour produire à moindre coût et augmenter les revenus. Mais il est certain qu'une meilleure maîtrise des étapes énergivores se traduit par une amélioration de la qualité des amandes et du beurre avec un effet positif sur les prix de vente et donc aussi sur les revenus.

Sur le plan environnemental, le bénéfice est de l'ordre 898 hectares de forêt épargnée dans la région de l'est du Burkina Faso, soit 60 kilotonnes d'équivalents $\mathrm{CO} 2$ évités, pour une production annuelle de 2706 tonnes de beurre. On peut aussi s'attendre à une amélioration de la santé des populations, moins exposées aux fumées des fours traditionnels.

Même si les chercheurs n'ont pas testé l'acceptabilité par les productrices de toutes les techniques et pratiques proposées, ils ont veillé à proposer du matériel simple, qui peut être fabriqué localement et promu par les programmes de développement. L'ensemble des équipements coûte au maximum 100000 FCFA, et les groupements de femmes peuvent l'acquérir progressivement, en fonction de leurs disponibilités financières.

\section{Analyse}

\section{Les agricultures familiales du monde}

Alors qu'elle opère parfois selon des modèles intensifiés (agro-chimie et motorisation) et spécialisés, l'agriculture familiale est également invoquée comme disposant de certaines caractéristiques pouvant apporter des solutions aux défis alimentaires, sociaux et environnementaux. En effet, la capacité du modèle agricole intensif à proposer des réponses adaptées aux défis contemporains est aujourd'hui largement questionnée, notamment en ce qui concerne le changement climatique, la sécurité alimentaire, la raréfaction des ressources fossiles, la prévention des maladies émergentes, mais aussi le développement de nouvelles sources énergétiques (comme la bioénergie) ou de modèles d'agriculture durable. La forme familiale de production aurait des qualités lui permettant de mettre en oeuvre un « autre modèle agricole », plus soucieux de l'environnement, plus riche en emplois, mieux intégré au sein des territoires. C'est ce questionnement qui est au centre de cette étude.

Dans ce cadre général, mais aussi en perspective de l'année internationale de l'Agriculture familiale décrétée par les Nations-Unies pour 2014, 1AFD, le MAE et le MAAF ont demandé au Cirad de préciser le contenu du concept d'agriculture familiale et sa pertinence par rapport aux autres catégories d'agricultures, et d'analyser les contributions économiques, sociales et environnementales des agricultures familiales ainsi que leur prise en compte par les politiques. Ce résumé rassemble les principales observations du rapport et présente les propositions opérationnelles pour une réelle reconnaissance de cette forme d'organisation de l'agriculture par les politiques publiques. Il conclut sur des perspectives d'approfondissement des réflexions.

Document téléchargeable sur le site du Cirad, www.cirad. fr : Accueil $>$ Publications \& ressources $>$ Edition $>$ Etudes et documents $>$ Les agricultures familiales du monde.

\section{Publications}

\section{0 recettes équilibrées avec les huiles et protéines végétales}

Proléa, filière française des huiles et protéines végétales, vient de publier une brochure de 28 pages proposant 20 recettes équilibrées avec les huiles et protéines végétales : tartare de betterave aux câpres, légumes croquants à l'aïoli, sucrine de feta à l'huile de noisette et poivron rouge, flan de lentilles corail et gambas marinées, parmentier de confit de canard aux mogettes de Vendée, rillettes de thon à la ciboulette sur son croûton à l'ail, tartare de langoustines et onctueux de petits pois à la menthe, etc. 
La brochure est téléchargeable gratuitement via le lien : http ://www.prolea.com/fileadmin/internet/fichiers/publications/Livre\%20recettes_28pages_BD.pdf.

\section{Quel futur pour notre alimentation?}

Dans un monde qui change profondément, le grand défi du $\mathrm{XXI}^{\mathrm{e}}$ siècle est de garantir aux hommes de l'eau et de la nourriture en quantité suffisante et de bonne qualité. Scientifiques, agriculteurs et industriels doivent gagner le pari de l'innovation pour répondre à cet enjeu. Comment seront produits, transformés et commercialisés nos aliments au cours des prochaines décennies? Au cours de ce voyage dans le futur, nous découvrons des agricultures «durablement productives », la place que pourraient occuper les organismes génétiquement modifiés, l'urgence à réduire les pertes de denrées alimentaires, le recours à des microorganismes et des algues, voire des insectes, la qualité sanitaire de notre alimentation qui ira en s'améliorant...

Quel futur pour notre alimentation?, par Pierre Feillet. 168 pages. Editions Quae, 2014.

\section{Au banquet de la nature - Alimentation, agriculture et politiques}

À l'origine d'une multitude de gestes et d'activités humaines, l'acte alimentaire - en tant que fait social total - imprime une marque profonde sur l'économie et les sociétés. À l'heure du village planétaire, ce livre incite le lecteur à scruter le système alimentaire mondial, promu par les multinationales de l'agrofourniture, de l'agroalimentaire ou de la grande distribution, mais aussi les modèles alternatifs, porté par le mouvement social, en vue de produire, d'échanger et de consommer des biens agricoles et alimentaires dans une perspective vertueuse.

Au banquet de la nature - Alimentation, agriculture et politiques, par Joseph Garnotel. 192 pages. Editions Quae, 2014.

\section{Agenda}

\section{Séminaire d'automne 2014 de la S.F.E.L.}

Le prochain séminaire d'Automne de la SFEL, intitulé «Oméga-3 à longue chaîne, de la production à la consommation : quelle balance aujourd'hui et demain?» se tiendra au FIAP (30 rue Cabanis, Paris 14e), salle Oslo, le vendredi 17 octobre 2014, de 14 h à 17 h.

Le programme (PDF) et le bulletin d'inscription (formulaire en ligne) sont disponibles via le site de la SFEL, www.sfel.asso.fr. Le nombre de place étant limité, il est conseillé de s'inscrire en ligne. Cette inscription est gratuite pour les adhérents de la SFEL à jour de leur cotisation. 